\title{
19. Hämophilie-Symposion
}

\section{Hamburg 1988}

\section{Herausgeber: G. Landbeck, R. Marx}

Verhandlungsberichte:

Neue Erkenntnisse zur ärztlichen Versorgung HIV-infizierter Hämophiler Substitutionstherapie-bedingte chronische Hepatitis

Diagnostik und Therapie der Hemmkörperhämophilie

Wissenschaftliche Leitung:

G. Landbeck, Hamburg

R. Marx, München

Moderatoren:

F. Deinhardt, München; K. Lechner, Wien; L. Bergmann, Frankfurt;

J. Bogner, München; H. Egli, Bonn; G. Landbeck, Hamburg;

W. Schramm, München; I. Scharrer, Frankfurt; H. Vinazzer, Linz;

L. Gürtler, München; Kl. Schimpf, Heidelberg; H. Rasche, Bremen;

M. Schulz, Hannover; P. Hellstern, Ludwigshafen;

H. Niessner, Wiener Neustadt; E. Wenzel, Homburg/Saar

Springer-Verlag Berlin Heidelberg New York London Paris Tokyo Hong Kong 


\section{Inhaltsverzeichnis}

Begrüßung und Einleitung . . . . . . . . . . . . . . . . . . . . . . 1

G. LANDBECK

Verleihung des Johann Lukas Schönlein-Preises 1988 . . . . . . . . . . . 3

G. LANDBECK

I. Neue Erkenntnisse zur ärztlichen Versorgung HIV-infizierter Hämophiler

1. Grundlagenreferate

Todesursachenstatistik und AIDS-Erkrankungen Hämophiler in der Bundesrepublik Deutschland 1988 . . . . . . . . . . . . . . . . . . 11

G. LANDBECK

Diskussion ....................... 19

Virologie der HIV-Infektion - Primär- und Verlaufsdiagnostik . . . . . . 22

L. Gürtler, J. Eberle, F. DeinhardT

Immunologie der HIV-Infektion - Primär- und Verlaufsdiagnostik . . . . 31

L. Bergmann, P. Hechler, E. B. Helm, P. S. Mitrou

Diskussion ....................... 41

Interventionstherapiestudien bei HIV-Infektion . . . . . . . . . . . . . 48

J. R. Bogner, A. Matuschke, B. Heinrich, H. S. Füessl, F.-D. Goebel

Diskussion ....................... 54

2. Spontanverlauf der HIV-Infektion

Verlaufsbeobachtung der HIV-Infektion bei Patienten mit hämorrhagischen

Diathesen .................... 6 61

F. Störkel, E. Aygören, L. Bergmann, H. W. Dörr, E. B. Helm, W. Preiser, A. Werner, I. Scharrer

Diskussion ..................... 66 
Partielle Verbesserung immunologischer in-vitro-Parameter bei HIV-infizierten Hämophilie-Patienten während eines Überwachungszeitraumes von mindestens zwei Jahren

V. Daniel, R. Weimer, K. Schimpf, G. Opelz

Spontane und Mitogen-stimulierte B-Zell-Differenzierung bei HIVinfizierten Hämophilie-Patienten . . . . . . . . . . . . . . . . . . . . . . 72

R. Weimer, T. Schweighoffer, V. Daniel, K. Schimpf, G. Opelz

Diskussion ....................... . . 79

Klinischer Verlauf der HIV-1-Infektion bei 51 Patienten mit Hämophilie $\quad$. 81

U. Brunkhorst, M. Barthels, B. Tschechne, I. Schedel, H. Deicher

Korrelation von immunologischen und virologischen Parametern zum

Krankheitsverlauf bei Hämophilie-Patienten mit HIV-1-Infektion . . . . . 86

B. Tschechne, M. Barthels, U. Brunkhorst, I. Schedel, H. Deicher

Diskussion ......................... 90

Verlaufsbeobachtungen zur HIV-Infektion hämophiler Kinder und

Jugendlicher

R. Bialek, N. Wagner, H. Radinger, M. Becker, H.-H. Brackmann,

D. NIESE, K. E. SCHNEWEIS

Diskussion ....................... . . 99

Nervensystembeteiligung bei HIV-infizierten Hämophilen . . . . . . . . . 101

R.-R. Riedel, D. Niese, H.-H. Brackmann, P. Clarenbach

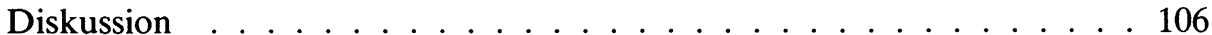

HIV-Antigen bei HIV-Antikörper-positiven hämophilen Kindern und Jugendlichen der Universitäts-Kinderklinik Graz . . . . . . . . . . . . . . 108

W. Zenz, W. Muntean, M. Wilders-Truschnig

Klinische, hämatologische und immunologische Parameter von 6 Anti-HIVpositiven Hämophilen . . . . . . . . . . . . . . . . . . . . . . . . . . . . 111

B. Zieger, W. Baden, H. Thaiss, A. H. Sutor, W. Künzer

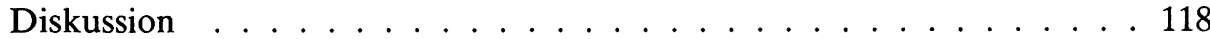

Kaposi-Sarkom - ein autoptisch gesicherter Zufallsbefund bei einem HIV-infizierten Jugendlichen mit Hämophilie A . . . . . . . . . . . . . . 119

U. NowaK-Görtl, W. Kreuz, S. Falk, A. Groll, H. Wolff, K. Hübner

Diskussion ...................... 125 


\section{Interventionstherapeutische Studien}

Immunglobulintherapie bei 10 HIV-AK-positiven Hämophilie-Patienten 129

E. Aygören, F. STÖRKEL, V. HACH-Wunderle, I. SChARRER

Immunglobulintherapie bei HIV-infizierten Hämophilen - klinische und immunologische Ergebnisse . . . . . . . . . . . . . . . . . 134

U. Wintergerst, C. Brückmann, K. Auberger, S. Gandenberger, H.-J. Klose, K. KöHLer-Vajta, K. Neumann, C. Rosendahl,

B. H. BELOHRADSKY

Diskussion

Polyradikulitis bei HIV-Manifestation bei einem Hämophilen . . . . . . . 143

D. Wesemeyer, H. Duscha, K. H. Zurborn, H. D. Bruhn

Diskussion

Retrovir-Behandlung von symptomatischen HIV-1-Antikörper-positiven Hämophilen

H. Hartl, I. Schwarzinger, Ch. Stain, I. Pabinger, K. Lechner

Diskussion

Schwere Immunthrombozytopenie bei einem HIV-1-positiven Hämophilie A-Patienten

W. BAden, J. Forster, H. SCHNeIder, B. Zieger, A. SUtor

Kontinuierliche Immunglobulintherapie in der Behandlung der

Thrombopenie bei Hämophilie-Patienten mit nachgewiesenen HIV-

Antikörpern . . . . . . . . . . . . . . . . . . . . . 158

H.-H. BrackmanN, B. v. Loo, H. EgLI, D. Niese, B. KampS

Hochdosierte Gabe von Immunglobulinen (IVIG) zur Behandlung passagerer blutungsgefährdeter Thrombozytopenien bei einem HIV-1-infizierten Hämophilie-Patienten . . . . . . . . . . . . . . 163

B. Tschechne, M. Barthels, U. BRUnKhorst, I. Schedel, H. Bock, H. DEICHER

Behandlung der HIV-assoziierten Thrombozytopenie mit Anti-D . . . . 167 U. WOLF

Diskussion 
4. Einfluß der Substitutionstherapie auf den Infektionsverlauf

Die perioperative Substitutionsbehandlung und Überwachung Anti-HIV (Human Immunodeficiency Virus)-positiver Hämophiler:

Drei Fallbeispiele . . . . . . . . . . . . . . . . . . . . . . . 177

G. Leipnitz, M. Köhler, G. Pindur, B. Rodemer, E. Wenzel

Diskussion

Das Auftreten von AIDS bzw. ARC oder Helferzellenmangel oder

Thrombopenie oder IgA-Erhöhungen in Abhängigkeit vom Konzentratverbrauch bei Hämophilie-Patienten mit nachgewiesenen HIV-Antikörpern

H.-H. Brackmann, B. v. Loo, D. Niese, B. Kamps, P. Euler,

U. HAMmerstein, H. Egli

Diskussion

\section{Infektionssicherheit kommerzieller Faktor VIII- und Faktor IX-Konzentrate}

Infektionssicherheit kommerzieller Faktor VIII- und Faktor IX-Konzentrate 197 W. SСHRAMm

Bericht über zwei laufende prospektive klinische Studien zur Virussicherheit von Gerinnungsfaktorenkonzentraten

K. SCHIMPF, P. M. MANNUCCI

Diskussion 206

Der Nachweis von Hepatitis B, NANB und HIV-Antikörpern bei ausschließlicher Verwendung zugelassener oder in klinischer Prüfung befindlicher virusinaktivierter Gerinnungskonzentrate . . . . . . . . 207 H.-H. Brackmann, H. Egli, B. v. Loo, P. Euler, G. Clauss

Diskussion . . . . . . . . . . . . . . . . . 212

Immunologischer Status und negative HIV-1- und HIV-2-Serologie nach Substitution mit virusinaktivierten Faktor VIII-Präparaten seit 1980 . . . . 214

W. Kreuz, B. Krackhardt, U. Ebener, A. Werner, H. v. Briesen, U. NOWAK-GöTtL, B. Wegerich, S. WeHner, R. KurTh, B. Kornhuber

Diskussion 220

In-vivo- und ex-vivo-Untersuchungen eines neuen monoklonal gereinigten F. VIII-Konzentrates . . . . . . . . . . . . . . . . . . 221

I. Scharrer, Z. Vigh, E. Aygören, F. STÖRKel, V. Hach-Wunderle

Diskussion 


\section{Substitutionstherapie-bedingte chronische Hepatitis}

Substitutionstherapie-bedingte chronische Hepatitis . . . . . . . . . 231

M. SCHULZ

Diskussion . . . . . . . . . . . . . . . . . . 241

III. Diagnostik und Therapie der Hemmkörperhämophilie

Allgemeine Grundlagen der Hemmkörperbildung . . . . . . . . . . 245

Chr. Mannhalter

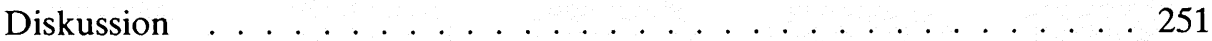

Behandlungsstrategien bei Patienten mit Hemmkörperhämophilie . . . . 253 W. SPEISER

Induzierte Immuntoleranz in der Behandlung von Hämophilie-Patienten mit Hemmkörpern. Ergebnisse aus 12 Jahren . . . . . . . . . . . 258 H.-H. BrackmanN

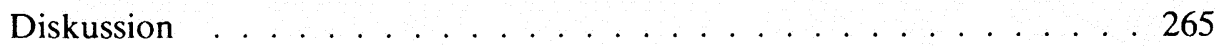

Behandlung der Hemmkörperhämophilie im Kindesalter . . . . . . 267

W. KREUZ, B. KRACKHARDT, I. SCHARRER

Diskussion . . . . . . . . . . . . . . . . . 275

Bestimmung von Faktor VIII-Inhibitoren mit der „Bethesda-Methode“ mit einem Einstufentest und einem chromogenen Assay zur Bestimmung der FVIII:C-Residualaktivität . . . . . . . . . . . . 276

P. Hellstern, D. Levi, G. Pindur, M. Köhler, E. Wenzel

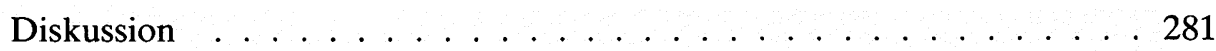

Therapie der Hemmkörperhämophilie: Erste Versuche mit dem Malmö

(Nilsson)-Protokoll . . . . . . . . . . . . . . . . . . . . . 283

E. Lechler, B. Roth, A. Fuchshuber, R. DReyer

Diskussion . . . . . . . . . . . . . . . . . . . . 289

IV. Freie Vorträge

Faktor VII-Verminderung - ein Zufallsbefund? . . . . . . . . . . . 293

K. HASLER, M. MaIer

Diskussion . . . . . . . . . . . . . . . . . . . . 296

Protein C-Gerinnungstest: Einfluß verschiedener PTT-Reagenzien auf die Eichgerade . . . . . . . . . . . . . . . . . . . . . 298

K.-H. Beck, B. PicARD-WILlems, I. ScharReR 


\title{
Interventionstherapiestudien bei HIV-Infektion
}

\author{
J. R. Bogner, A. Matuschke, B. Heinrich, H. S. Füessl, F.-D. Goebel \\ (München)
}

Zunächst stellt sich die Frage, welche Eigenschaften eine ideale antiretrovirale Substanz aufweisen müßte. Die Antworten klingen einfach. Folgendes müßte gewährleistet sein: Eine effektive Hemmung der Virusreplikation, nicht nur in vitro, sondern auch in vivo; eine Toxizität, die auch in einer Langzeittherapie nicht limitierend ist; eine maximale therapeutische Breite; Fehlen von Resistenzentwicklung; günstige pharmakokinetische Eigenschaften bezüglich Passage der Blut-Hirn-Schranke, Plasmahalbwertszeit und Bioverfügbarkeit bei oraler Applikation. Nicht zuletzt ist hinsichtlich des Langzeiteinsatzes idealerweise eine kostengünstige Substanz wünschenswert [1].

Eine Aufstellung über die Selektivität von antiretroviralen Substanzen haben De ClercQ et al. zusammengestellt [2]. Ein Selektivitätsindex wurde gebildet aus dem Quotienten von Zytotoxizitätsdosis in der Zellkultur $\mathrm{CD}_{50}$ und in vitroEffektivitätsdosis $\mathrm{ED}_{50}$. Hierbei wurde ein günstiger Selektivitätsindex gefunden einerseits für verschiedene Dideoxynukleotide wie z.B. Azidothymidin und Dideoxycytidin, andererseits aber aber auch für Substanzen, die hauptsächlich Wirksamkeit an der Zelloberfläche von Zielzellen entfalten, wie z.B. Dextransulfat und Heparin. Substanzen, die klassischerweise als Hemmstoffe der Reversen Transkriptase (RT) untersucht wurden, wie z.B. Suramin und Ribavirin, zeigen bei relativ hoher Toxizität in dieser Untersuchung einen relativ ungünstigen Selektivitätsindex.

\section{Erfolgsparameter für Studien mit antiretroviralen Substanzen}

Die Effektivität einer antiretroviralen Substanz ist bei geeigneter Technik in vitro unproblematischer zu beurteilen als in einer klinischen Studie. Hier gelten als Erfolgsparameter:

- eine Verringerung der RT Aktivität

- eine Verminderung des zytopathischen Effekts von HIV

- und die Inhibition der Syncytienbildung.

Die Beurteilung der klinischen Effektivität einer antiviralen Substanz muß sich am natürlichen Verlauf der Infektion, an der Auswirkung auf den klinischen Zustand des Patienten, sowie an immunologischen und virologischen Parametern orientieren. 
Je früher im Verlauf der HIV-Infektion eine Therapie klinisch getestet werden soll, desto schwieriger ist die Erfolgsbeurteilung, da die mittlere Inkubationszeit mit 8 bis 10 Jahren geschätzt wird.

Wenn man für HIV-Infizierte ohne bekanntes Infektionsdatum eine jährliche Progressionsrate zum AIDS-Vollbild von $7-10 \%$ veranschlagen darf, so bedarf es sehr hoher Patientenzahlen und langer Behandlungsjahre um eine Beeinflussung des natürlichen Verlaufs statistisch zu sichern.

Bei symptomatischen Patienten der Diagnosegruppe CDC III und IV-A kann neben der Beurteilung des subjektiven Wohlbefindens der Patienten auch auf klinisch objektivierbare Befunde zurückgegriffen werden: Verlauf des Körpergewichtes, Häufigkeit von HIV-assoziierten Symptomen und Häufigkeit sowie Schwere der unter Therapie auftretenden opportunistischen Infektionen.

An virologischen Erfolgsparametern haben sich die Bestimmung von p24-Antigenspiegeln und die Anzüchtbarkeit von HIV in der Zellkultur als übliche Methoden herauskristallisiert. Über die antiretrovirale Auswirkung der studierten Substanz auf die Virusreplikation in Gewebsmakrophagen und anderen Körperzellen wird allerdings mit diesen Methoden keine Aussage gemacht.

An immunologischen Parametern wird bisher üblicherweise der Verlauf der CD4-Zellen in Prozent oder absolut verwendet. Solange die Pathogenese der CD4-Zell-Depletion nicht ausreichend geklärt ist, besteht die Frage, ob nicht auch andere pathogenetisch wichtige Parameter zur Beurteilung einer Therapie gefunden werden müssen. Ein neuer Ansatzpunkt hierzu wäre, die Zahl der bereits im frühen Stadium vermehrten aktivierten T-Lymphozyten heranzuziehen.

Außerdem können indirekte, unspezifische Parameter als Maß für die Aktivierung der zellulären Immunität wie Neopterin und Beta-2-Mikroglobulin für die Verlaufs- und Effektivitätsbeurteilung eine größere Bedeutung gewinnen [3].

Die Messung der Hautreaktivität auf Recall-Antigene ist bisher in allen Studien gebräuchlich. Die Aussage hierdurch stößt aber insofern auf Grenzen, da einerseits mit Boosterreaktionen gerechnet werden muß, andererseits eine Vergleichbarkeit der Ergebnisse strenggenommen nur gewährleistet ist, wenn sie von demselben Untersucher abgelesen werden.

\section{Angriffspunkte im HIV-Replikationszyklus}

Neben den besonders gründlich untersuchten Hemmstoffen der RT und DNASynthese-Inhibitoren sind folgende Angriffspunkte von Interesse. Auf der Rezeptorebene an der Zelloberfläche kann die Adhäsion von HIV-Partikeln an den CD4-Rezeptor mehr oder weniger spezifisch inhibiert werden, z.B. durch Substanzen wie AL721, Dextransulfat, Peptid T und monoklonale CD4-Antikörper. Löslichen CD4-Antikörpern und gereinigten neutralisierenden Antikörpern (Anti-GP120, Anti-p24) wird die neutralisierende Wirkung noch vor der VirusAdhäsion zugeschrieben [3a].

Auf der Ebene des „uncoating“ der viralen RNA wird Interferonen eine hemmende Wirkung zugeschrieben. 
Auf der Ebene der reversen Transkription und DNA-Komplementierung können RT-Hemmstoffe und Nukleotide, die zum Kettenabbruch der DNA-Synthese führen, zur Wirkung kommen. Hier seien die klassischen RT-Inhibitoren Suramin, HPA 23, Ribavirin und Foscarnet einerseits, und die Gruppe der Dideoxynukleotide andererseits genannt.

Weitere Wirkungsmechanismen sind auf der Ebene der Translation und des „virus assembly“ möglich.

Hier wurde in letzter Zeit die Aufmerksamkeit auf Protease-Inhibitoren und Glykosylierungs-Hemmstoffe gelenkt. Für d-Penicillamin wird beispielsweise ebenfalls eine diesbezügliche Aktivität diskutiert [4].

Von besonderem theoretischen Interesse sind natürlich Therapiestudien, in welchen an zwei unterschiedlichen Zielpunkten im Replikationszyklus angesetzt wird, z.B. die Kombination von AZT mit Interferon.

\section{Studien mit antiretroviralen Substanzen}

Für niedermolekulares Dextransulfat sind als Wirkungsmechanismen eine Blokkade der Virusadhäsion an den CD4-Rezeptor und eine Verhinderung der Syncytienbildung aus in-vitro-Studien bekannt. Bei einer 8 wöchigen Pilotstudie an 34 Patienten mit ARC und AIDS kam es zu einem leichten CD4-Anstieg ohne signifikante Änderung der p24-Antigen-Spiegel [5]. An Nebenwirkungen traten vor allem Hepatotoxizität und Leukopenie auf. Weitere Studienergebnisse werden mit Spannung erwartet.

Die Abkürzung $A L 721$ steht für ,active lipids“. Das Mischungsverhältnis von Triglyceriden, Phosphatidylcholin und Phosphatidylethanolamin ist 7:2:1. Es wirkt über Membranfluidisierung und Oberflächeneffekte, die bisher nicht eindeutig charakterisiert sind. Ergebnisse aus vorliegenden Studien sind eher widersprüchlich [6, 7]: Einerseits: Hemmung der RT-Aktivität und subjektive Besserung. Andererseits: Abnahme der CD4-Zellen und Zunahme von opportunistischen Infekten bei AIDS-Vollbild-Patienten. Immerhin gilt die Substanz als nebenwirkungsfrei und wird derzeit in multizentrischen Studien an Patienten ohne AIDS-Vollbild getestet.

Von Peptid $T$ wird als Wirkungsmechanismus ebenfalls eine VirusrezeptorInteraktion postuliert [8]. Es wurde zwar über eine Zunahme von Helferzellen und Besserung von HIV-Enzephalitis berichtet, virologische Daten fehlen jedoch noch, so daß ein Wirkungsnachweis noch nicht als gesichert gilt.

CD4-Antikörper können durch ihre spezifische Reaktion mit HIV-Oberflächenprotein eine vollständige Blockade der Virusbindung an zellständiges CD4Protein der Zielzellen bewirken. Allerdings hat der CD4-Rezeptor eine physiologische Funktion in der Zellinteraktion von Lymphozyten. An Mäusen wurde eine Blockade der Helferzellfunktion gezeigt [9]. Eine Beurteilung als Therapeutikum steht derzeit noch aus. Lösliches CD4-Antigen kann spezifisch an gp120 von HIV binden und damit eine Neutralisierung vor der Virusadhäsion einerseits und eine Inhibition der Syncytienbildung andererseits bewirken. Es besteht aber auch hier die Frage, ob nicht physiologische Immunfunktionen gehemmt werden [9a]. 
Für alpha-Interferon gilt die therapeutische Wirksamkeit für die Rückbildung des Kaposi-Sarkoms in einem gewissen Prozentsatz der Behandelten als gesichert [10]. Außerdem kommt es zu einer Abnahme von p24-Antigen-Spiegeln. Allerdings handelt es sich um eine nebenwirkungsreiche Therapie. In weiteren Studien wurde die Indikation für alpha-Interferon in jüngster Zeit auf HIV-Infizierte ohne Kaposi-Sarkom ausgedehnt. Die Möglichkeit einer oralen Applikation wird gegenwärtig überprüft.

Foscarnet hemmt virale DNA-Polymerasen, nicht nur von HIV. Es kommt zwar zu einer RT-Hemmung in vivo, die Hauptschwierigkeiten mit der Substanz liegen jedoch in der mangelnden enteralen Resorption und im Nebenwirkungsreichtum. Neben Niereninsuffizienz und Anämie traten auch subjektive Nebenwirkungen auf [11].

Für Ribavirin ist bisher ein eindeutiger Wirkungsnachweis in vivo nicht erbracht. Bei einer ersten placebokontrollierten Studie traten bei Patienten der Placebogruppe mehr opportunistische Infektionen auf als in der Verum-Gruppe. Allerdings wurden Zweifel an dieser Untersuchung geäußert, da die Patienten der Placebogruppe offensichtlich bei Studienbeginn einen schlechteren immunologischen Ausgangsstatus aufwiesen [12].

Neben Azidothymidin gibt es auch unter den Purinen 2,3-Deoxynukleotide, das Dideoxyadenosin und Dideoxyinosin. Mit ersterem wurde im Februar 88 eine Phase I Studie begonnen.

Ergebnisse mit Dideoxycytidin deuten auf eine gute Wirksamkeit hin: ein Abfall von p24-Antigen und ein vorübergehender Anstieg der Helferzellen [13]. Allerdings zeigte sich bei einigen Patienten, die länger als 6 Wochen behandelt wurden, eine periphere sensomotorische Neuropathie. Auch Fieber, Abgeschlagenheit, Exanthem und Schleimhautulzerationen wurden als Nebenwirkungen beobachtet. Phase II Studien werden nur in Kombination mit AZT durchgeführt.

In einer Pilotstudie mit dieser Kombination $(A Z T+d d C)$ über 9 Wochen mit wöchentlichem Wechsel der beiden Substanzen kam es zu einer CD4-Zunahme und p24-Antigen-Abnahme. Ein Patient, der bereits zuvor ddC erhalten hatte, entwickelte ebenfalls eine Neuropathie. In weiteren Studien wird wöchentlicher Substanzwechsel versus vierwöchentlichen Wechsel getestet.

Neben der genannten Kombination von AZT und ddC wird AZT in weiteren Kombinationen getestet, z.B. mit Interferon, Acyclovir, Granulozyten-Makrophagen colony stimulating factor (GM-CSF) zur Verhinderung der Myelotoxizität, und mit Interleukin.

Die Kombination von AZT mit alpha-Interferon wurde nach den bisher vorliegenden Ergebnissen zwar relativ gut vertragen, ist aber insgesamt eher zurückhaltend bewertet worden. Fischl berichtet über enttäuschende virologische Ergebnisse [14], ORHOLм [15] über verstärkte Myelotoxizität und BRATZKe über das Fehlen einer verbesserten Effektivität gegenüber der Monotherapie mit Interferon [16]. Interessant ist allerdings, daß durch die Kombination von AZT und Interferon alpha bei Mäusen eine retrovirale Neuinfektion verhindert werden kann [17].

Als interessant wurde auch die Kombination von AZT mit Acyclovir angesehen. Nach den vorliegenden Ergebnissen kann als gesichert gelten, daß diese Kombination nicht zu einer veränderten Pharmakokinetik der Einzelsubstanzen führt. Eine verbesserte immunologische oder virologische Wirksamkeit dieser 
Kombination bezüglich der HIV-Infektion wurde von keiner Gruppe schlüssig nachgewiesen. Allerdings kann aus der doppelblinden, placebokontrollierten europäischen Multicenterstudie immerhin vorläufig gefolgert werden, daß nach 48 Behandlungswochen weniger Herpes-Virusinfektionen und weniger opportunistische Infektionen bei Kombinationsbehandlung auftraten als bei Monotherapie mit AZT [18].

Weitere Studien mit Azidothymidin beziehen sich im wesentlichen auf eine erweiterte Indikationsstellung der antiretroviralen Therapie und eine differenziertere Dosierung des Medikaments. An Kaposi-Patienten wird die 8stündliche versus 4stündliche Applikation untersucht, bei HIV-dementia-complex werden $1000 \mathrm{mg} / \mathrm{d}$ versus $2000 \mathrm{mg} / \mathrm{d}$ untersucht. Bei Hämophilen ohne Immundefekt wird eine doppelblinde placebokontrollierte Studie mit einer täglichen Dosierung von $1500 \mathrm{mg}$ durchgeführt. Über die kontinuierliche intravenöse AZT-Therapie bei 21 Kindern zwischen 14 Monaten und 12 Jahren wurde kürzlich im New England Journal of Medicine berichtet [19]: Bei einer Behandlungsdauer von bis zu 50 Wochen konnte eine deutliche Besserung der Intelligenzleistungen und eine Verminderung der opportunistischen Infektionen gezeigt werden. Allerdings traten bei immerhin 10 Patienten lokale oder systemische bakterielle Komplikationen im Zusammenhang mit dem für die Therapie verwendeten Katheter auf.

Eine nicht kausale, nicht direkt im Replikationszyklus von HIV ansetzende Therapie, stellt die Therapie mit Immunglobulinen dar. Es sei aber darauf hingewiesen, daß bisher an pädiatrischen Patienten dennoch über einen Anstieg von CD4-Zellen, eine gesteigerte Mitogen-Reaktivität von Lymphozyten und einen Rückgang der Lymphadenopathie berichtet wurde [20]. Außerdem kann bei Patienten mit B-Zell-Dysfunktion eine Verminderung der febrilen Episoden, ein Rückgang der bakteriellen Infektionen und opportunistischen Infekte erzielt werden [21].

\section{Literatur}

1. Yarchoan R, Broder S (1987) Strategies for the pharmacologic intervention against HTLV III/LAV. In: Broder S (ed) AIDS: Modern concepts and therapeutic challenges. Dekker, New York, pp 335-360

2. DeClercq E (1988) Antiviral treatment for HIV-infection. In: AIDS/HIV experimental treatment directory $2 ; 1-6 \mathrm{I} / 1988$

3. Bogner J.R. et al (1988) Serum neopterin levels as predictor of AIDS. Klin Wochenschr 66:1015-1018, 1988

3a. Jackson GG et al. (1988) Passive imunoneutralization of HIV p24 antigenemia in patients with advanced AIDS by infusion of human plasma. Abstract 3064, IV. Int. Conf on AIDS, Stockholm

4. Paruti DM et al. (1987) D-penicillamine (DPA) treatment for lymphadenopathy syndrome (LAS) and AIDS-related complex. Abstract TP.220; III. Int. Conf. on AIDS, Washington

5. Mitsuya $\mathrm{H}$ et al. (1988) Dextran sulfate suppression of viruses in the HIV family. Inhibition of virion binding to $\mathrm{CD} 4^{+}$cells. Science $240: 646-649$

6. Yust I et al. (1988) Reduction of circulation HIV-antigen in seropositive patients after treatment with AL 721, Abstract 3530, IV. Int. Conf. on AIDS, Stockholm

7. Goebel F-D et al. (1988) Clinical findings after administration of lipids in AIDS. A pilot study. Abstract 3531, IV. Int. Conf. on AIDS, Stockholm 
8. Pert CB, Ruff MR (1986) Peptide T(4-8)-a pentapeptide sequence in the AIDS virus envelope which blocks infectivity is essentially conserved across 9 isolates. Clin Neuropathol 9:482-484

9. Carteron NL et al. (1988) Induction of immune tolerance during administration of monoclonal antibody to L3T4 does not depend on depletion of L3T4+ cells. J Immunol 140:713-716

9a. Weiss RA (1988) Receptor molecule blocks HIV. Nature 331:15

10. Abrams DI et al. (1987) Alpha interferon therapy of AIDS-associated Kaposi's sarcoma. Semin Oncol 14:527-531

11. Bergdahl S et al. (1988) Antiviral effect against HIV in patients with AIDS-related complex given intermittent i.v. foscarnet. Abstract 3588, IV. Int. Conf. on AIDS, Stockholm

12. Mansell PWA, Heseltine PNR et al. (1987) Ribavirin delays progression of the lymphadenopathy syndrome (LAS) to the acquired immunodeficiency syndrome (AIDS), Abstract T.8.5., III. Int. Conf. on AIDS, Washington

13. Yarchoan R et al. (1988) Phase I studies of 2'3'-dideoxycytidine in severe human immunodeficiency virus infection as a single agent and alternating with zidovudine (AZT). Lancet I:76-81

14. Fischl M et al. (1988) Phase I study of interferon alpha and AZT in patients with AIDS-related Kaposi's sarcoma. Abstract 3133, IV. Int. Conf. on AIDS; Stockholm

15. Orholm M et al. (1988) Effect on serum p24 antigen of a combination therapy with low dose interferon and zidovudine. Abstract 3626, IV. Int. Conf. on AIDS, Stockholm

16. Bratzke B et al. (1988) Combination of interferon (ra2a-IFN) and zidovudine for the therapy of HIV-associated Kaposi-sarcoma (KS). Abstract 3631, IV. Int. Conf. on AIDS, Stockholm

17. Ruprecht RM et al. (1988) Postexposure therapy with azidothymidin and interferon alpha prevents retroviral infection. Abstract 3632, IV. Int. Conf. on AIDS, Stockholm

18. Seligman M (1988) Zidovudine, plus or minus acyclovir, in the treatment of AIDS patients post-opportunistic infection. Wellcome-Satelliten-Symposium, Stockholm

19. Pizzo PA et al. (1988) Effect of continuous intravenous infusion of zidovudine (AZT) in children with symptomatic HIV infection. N Engl J Med 319:889-896

20. Calvelli TA, Rubinstein A (1986) Intravenous gamma globulin in infant acquired immunodeficiency syndrome. Pediatr Infect Dis 5:207-210

21. Brunkhorst $U$ et al. (1988) I.v. gammaglobulin in treatment of symptomytic HIV-1 infection. Abstract 7251, IV. Int. Conf. on AIDS, Stockholm 